\title{
Intracellular delivery of 2-deoxy-D-glucose into tumor cells by long-term cultivation and through swelling-activated pathways: Implications for radiation treatment
}

\author{
CHOLPON S. DJUZENOVA ${ }^{1}$, JULIA KRASNYANSKA ${ }^{1}$, MARTIN KIESEL ${ }^{2}$, LAVINIA STINGL ${ }^{1}$, \\ ULRICH ZIMMERMANN $^{2}$, MICHAEL FLENTJE ${ }^{1}$ and VLADIMIR L. SUKHORUKOV ${ }^{2}$ \\ ${ }^{1}$ Klinik für Strahlentherapie, Universität Würzburg, Josef-Schneider-Strasse 11, 97080 Würzburg; \\ ${ }^{2}$ Lehrstuhl für Biotechnologie, Universität Würzburg, Biozentrum, Am Hubland, 97074 Würzburg, Germany
}

Received November 17, 2008; Accepted March 20, 2009

DOI: $10.3892 / \mathrm{mmr} 00000149$

\begin{abstract}
Deoxy-D-glucose (2DG), a well-known inhibitor of anaerobic glycolysis, is expected to exert cytotoxic and radiosensitizing effects. In order to test this hypothesis, the response of four tumor cell lines (U87-MG, GaMG, A549 and HT1080) to 2DG was analyzed for cell proliferation, changes in cell volume and nucleus size, as well as for radiation-induced DNA fragmentation, measured by the alkaline Comet assay. Two methods were used for loading cells with 2DG. The long-term method included cell cultivation in the presence of $5 \mathrm{mM} 2 \mathrm{DG}$ for $24 \mathrm{~h}$, while rapid intracellular delivery of 2DG was achieved by exposing the cells for $20 \mathrm{~min}$ to a hypotonic solution containing $100 \mathrm{mM}$ 2DG. Irrespective of the loading method, 2DG inhibited the growth of HT1080 and A549 cells. In contrast, two glioblastoma lines (U87 and GaMG) were resistant to 2DG. In three of the four cell lines (all except HT1080), long-term treatment with 2DG reduced radiation-induced DNA fragmentation in conjunction with 2DG-mediated nucleus shrinkage (probably via chromatin condensation) in non-irradiated cells. Complementary volumetric experiments revealed the avid hypotonic uptake of 2DG by all tumor lines. Nonetheless, only HT1080 cells exhibited a significant increase in radiation-induced DNA fragmentation upon hypotonic loading with 2DG, associated with marked nucleus expansion in non-irradiated samples. Our data suggest that, dependant on cell type as well as on
\end{abstract}

Correspondence to: Dr Cholpon S. Djuzenova, Klinik für Strahlentherapie der Universität Würzburg, Josef-SchneiderStrasse 11, D-97080 Würzburg, Germany

E-mail: djuzenova_t@klinik.uni-wuerzburg.de

Abbreviations: 2DG, 2-deoxy-D-glucose; CGM, complete growth medium; FSC, forward scatter; IR, ionizing radiation; PI, propidium iodide; RVD, regulatory volume decrease; TM, Tail Moment

Key words: radiosensitization, Comet assay, regulatory volume decrease, hypotonic loading medium composition and tonicity, sugar treatment can induce the compaction or expansion of chromatin, thus decreasing or increasing radiation-induced DNA fragmentation. These results raise interesting questions for further studies on the mechanistic links between the sugar-modulated cell volume changes, chromatin structure and radiosensitivity of tumor and normal cells.

\section{Introduction}

Most cancer cells metabolize more glucose than their normal counterparts (1). This phenomenon is widely used in positron emission tomography for the localization of cancer tissues based on the preferential uptake of $\mathrm{F}^{18}$-labeled 2-deoxy-Dglucose (2DG). 2DG is a potent inhibitor of glucose metabolism (2). There is a body of evidence indicating that 2DG enhances the in vitro sensitivity of various cancer cells to ionizing radiation (IR) and cytostatic drugs (3-8). Due to its ability to mimic glucose deprivation in cancer cells, 2DG has been subject to clinical trials as an adjuvant to radiotherapy for gliomas $(9,10)$. Patients tolerate 2DG well, but the lack of insight into the mechanisms underlying its radio-/chemosensitization activity prevents the development of efficient 2DG-based therapies (11).

A recent in vitro study revealed the selective cytotoxicity of 2DG to hypoxic osteosarcoma cells (143b line) compared to an aerobic culture (12). 2DG also exerted significant cytotoxicity towards Chinese hamster V79 cells grown as multicellular spheroids under aerobic conditions (13). In animal studies, 2DG inhibited the growth of rat sarcoma (14). However, certain tumors that initially exhibit sensitivity to 2DG appear to become refractory to this sugar over time (11).

In contrast to the above observations, 2DG was reported to be incapable of killing murine fibrosarcoma and mammary adenocarcinoma hypoxic cells (15), and moreover conferred radioresistance to $S$. cerevisiae (16). There was also great heterogeneity in the 2DG-mediated alterations of radiation response among six different tumor cell lines in terms of the rate of DNA repair, micronuclei induction and apoptosis/ necrosis, among others (17). The authors of this prior study acknowledged that the prediction of cellular response to a combined IR-2DG treatment based on any radiobiological 
parameters might have severe limitations (15). In addition, 2DG would appear to be toxic in select tumor cell types growing under normoxia due to the inhibition of N-linked glycosylation, and not glycolysis (18).

The conflicting results reported in the literature prompted the present study, in which we explored the cytotoxicity and radiosensitizing ability of $2 \mathrm{DG}$ in a series of cell lines originated from different tumor entities. These included four human tumor cell lines: lung carcinoma A549, fibrosarcoma HT1080, and two glioblastoma lines, U87-MG and GaMG. Two different methods were used for the intracellular delivery of 2DG. In the first, tumor cells were incubated for $24 \mathrm{~h}$ in medium supplemented with $2 \mathrm{DG}$ at a clinically relevant concentration of $5 \mathrm{mM}$. The second loading method made use of 2DG-permeable pathways activated in tumor cell membranes by hypotonic swelling. Hypotonic loading was achieved by the 20-min exposure of cells to 100 -mOsm 2DGsubstituted medium. Efficient delivery of 2DG into tumor cells was verified by complementary time-resolved volumetric measurements, in which the membrane-impermeable disaccharide sucrose served as a reference for comparison to 2DG. Each tumor cell line (treated with 2DG, sucrose, IR, or combined sugar-IR exposure) was analyzed for proliferation rate, changes in cell size and cell cycle distribution. Radiationinduced DNA fragmentation was also assessed by the alkaline Comet assay.

\section{Materials and methods}

Cells. The human tumor cell line group included the glioblastomas U87-MG (U87) and GaMG, the lung carcinoma A549 and the fibrosarcoma HT1080. Cells were obtained from the American Type Culture Collection (ATCC; Manassas, VA) and were routinely cultured in complete growth medium (CGM) under standard conditions $\left(5 \% \mathrm{CO}_{2}\right.$ and $\left.37^{\circ} \mathrm{C}\right)$. Either MEM (U87 and GaMG) or DMEM (A549 and HT1080) was used as the CGM.

Hypotonic solutions and cell volumetry. Hypotonic sugarsubstituted solutions contained $100 \mathrm{mM}$ 2DG (or sucrose) as the major solute and $2 \mathrm{mM}$ HEPES-KOH ( $\mathrm{pH} 7.2$ ). Osmolality was determined cryoscopically (Osmomat 030, Gonotec, Berlin, Germany).

Cell volume changes were measured by videomicroscopy in a microfluidic chamber as previously described (19). Prior to the taking of measurements, cells suspended in isotonic CGM ( $\sim 300 \mathrm{mOsm})$ were injected into the chamber and allowed to adhere to the glass coverslip. Images of the cells were taken $1 \mathrm{~min}$ before and at various time intervals up to $25 \mathrm{~min}$ after the exchange of the medium using a videomicroscopic setup described elsewhere (19). Cross-sectional areas of the cells were determined with the ScionImage image analysis program (Scion, Frederick, MD). At each time interval, the volume V of individual cells was evaluated based on their cross-sectional area by assuming a spherical geometry. Cell volume values were normalized to the original isotonic volume $\left(v=\mathrm{V} / \mathrm{V}_{0}\right)$ and plotted versus time.

$X$-ray irradiation. Irradiation was performed at room temperature using a $6 \mathrm{MV}$ Siemens linear accelerator (Siemens, Concord, CA) at a dose rate of $2 \mathrm{~Gy} / \mathrm{min}$.
Cell counting and flow cytometry. Exponentially growing cells were seeded in duplicate for $24 \mathrm{~h}$, then exposed to sugar treatments $(5 \mathrm{mM})$ for the next $24 \mathrm{~h}$. Cells were counted with a Coulter Counter Z1 (Coulter Electronics, Krefeld, Germany).

For flow cytometry, cells were trypsinized, washed twice in PBS and resuspended in $0.5 \%$ saponin (Sigma S-2149) in PBS for $5 \mathrm{~min}$ (reviewed in ref. 20). Staining with propidium iodide (PI, Sigma P-4170, $25 \mu \mathrm{g} / \mathrm{ml}$ ) was performed in the presence of ribonuclease (Sigma R-5250, $25 \mu \mathrm{g} / \mathrm{ml}$ ). Forward scatter (FSC) and DNA distribution (PI-staining) were measured with the FACSCalibur (Becton-Dickinson, San Jose, CA).

Comet assay. Control and sugar-treated cells were trypsinized and re-suspended in sugar-free CGM, CGM supplemented with $5 \mathrm{mM}$ sugar or hypotonic (100 mOsm) sugar-substituted solution at a density of $\sim 4 \times 10^{6}$ cells $/ \mathrm{ml}$. Just before irradiation, the cells were embedded in a thin layer of agarose spread on glass microscope slides. The slides were placed on ice, subjected to X-irradiation, and then transferred immediately to ice-cold lysis buffer (reviewed in refs. 21 and 22).

The Comet assay was performed under alkaline conditions following a protocol reported elsewhere $(21,22)$. DNA fragmentation was quantified from the 'Tail Moment' (TM, given in arbitrary units, a.u.), defined as the product of the percentage of DNA in the comet tail and the tail length (23). The nuclear area based on the integrated intensity profile was also extracted from the Comet assay data.

Immunofluorescence staining. The primary antibodies used were rabbit polyclonal anti-aquaporin 1 (AQP1, Chemicon, Temecula, CA) and rabbit polyclonal anti-aquaporin 4 (AQP4, Santa Cruz Biotechnology, CA). Secondary species-specific antibodies were labeled with horseradish-peroxidase (Dako, Hamburg, Germany) or Alexa Fluor $488 \mathrm{~nm}$ and $594 \mathrm{~nm}$ (Molecular Probes, Eugene, OR) for Western blot analysis and immunofluorescence detection (24), respectively.

\section{Results}

Long-term effects of the sugars. Treatment of human breast cancer cells in vitro with $2 \mathrm{DG}$ results in the cessation of cell growth (25). To determine whether 2DG induces a similar effect in cell lines derived from other tumor entities, four human cancer cell lines were cultured for $24 \mathrm{~h}$ in CGM supplemented with $5 \mathrm{mM}$ 2DG. Control cells were grown in standard CGM or in CGM supplemented with $5 \mathrm{mM}$ sucrose. 2DG strongly inhibited the growth of HT1080 cells, without affecting the proliferation of GaMG cells (Table I). Although the growth rates of U87 and A549 cells also decreased slightly in the presence of 2DG, these effects did not reach statistical significance. Sucrose did not notably affect the growth of tumor cells.

Fig. 1 shows representative FSC distributions for four cell lines and the various sugar treatments. The unchanged FSC properties of U87 and GaMG cells suggest that neither 2DG nor sucrose altered cell size distribution in the glioblastoma lines compared to the corresponding controls (upper row).

2DG significantly decreased the cell size of HT1080 cells, evidenced by the strong shift in the FSC peak (Fig. 1). Unlike 2DG, sucrose did not cause any notable changes in the FSC histograms of HT1080 cells. Interestingly, 2DG caused the 
Table I. Relative changes ${ }^{\mathrm{a}}$ in cell growth after long-term incubation ${ }^{\mathrm{b}}(5 \mathrm{mM}, 24 \mathrm{~h})$ and after a short-term hypotonic ${ }^{\mathrm{c}}\left(100 \mathrm{mM}^{\mathrm{m}}\right.$, $20 \mathrm{~min}$ ) treatment of tumor cells with $2 \mathrm{DG}$ or sucrose.

\begin{tabular}{llcrr}
\hline & \multicolumn{3}{c}{ Sugar treatments } \\
\cline { 2 - 5 } Cell line & \multicolumn{2}{c}{$2 \mathrm{DG}$} & \multicolumn{2}{c}{ Sucrose } \\
\cline { 2 - 5 } & $5 \mathrm{mM}, 24 \mathrm{~h}$ & $100 \mathrm{mM}, 20 \mathrm{~min}$ & $5 \mathrm{mM}, 24 \mathrm{~h}$ & $100 \mathrm{mM}, 20 \mathrm{~min}$ \\
\hline U87 & $0.85 \pm 0.08$ & $1.02 \pm 0.21$ & $1.14 \pm 0.08$ & $1.02 \pm 0.24$ \\
A549 & $0.77 \pm 0.01$ & $0.76 \pm 0.20$ & $1.09 \pm 0.14$ & $1.04 \pm 0.30$ \\
HT 1080 & $0.49 \pm 0.04$ & $0.80 \pm 0.04$ & $0.99 \pm 0.05$ & $0.94 \pm 0.35$ \\
GaMG & $1.09 \pm 0.16$ & $1.30 \pm 0.61$ & $1.42 \pm 0.07$ & $0.88 \pm 0.38$ \\
\hline
\end{tabular}

${ }^{\mathrm{a}}$ Data are the normalized means $( \pm \mathrm{SE})$ of at least three independent experiments. ${ }^{\mathrm{b}}$ Cells were incubated for $24 \mathrm{~h}$ in CGM containing $5 \mathrm{mM}$ of 2-DG or sucrose. The cells were then counted, and the data were normalized to the corresponding isotonic controls. ${ }^{\mathrm{c} C e l l s}$ were treated for $\sim 20 \mathrm{~min}$ in a hypotonic sugar medium. Subsequently, the cells were transferred to CGM at the same final cell density of $2 \times 10^{4}$ cells $/ \mathrm{ml}$ and cultured for 3 days under standard conditions. The cells were then counted, and the data were normalized to the corresponding isotonic controls.

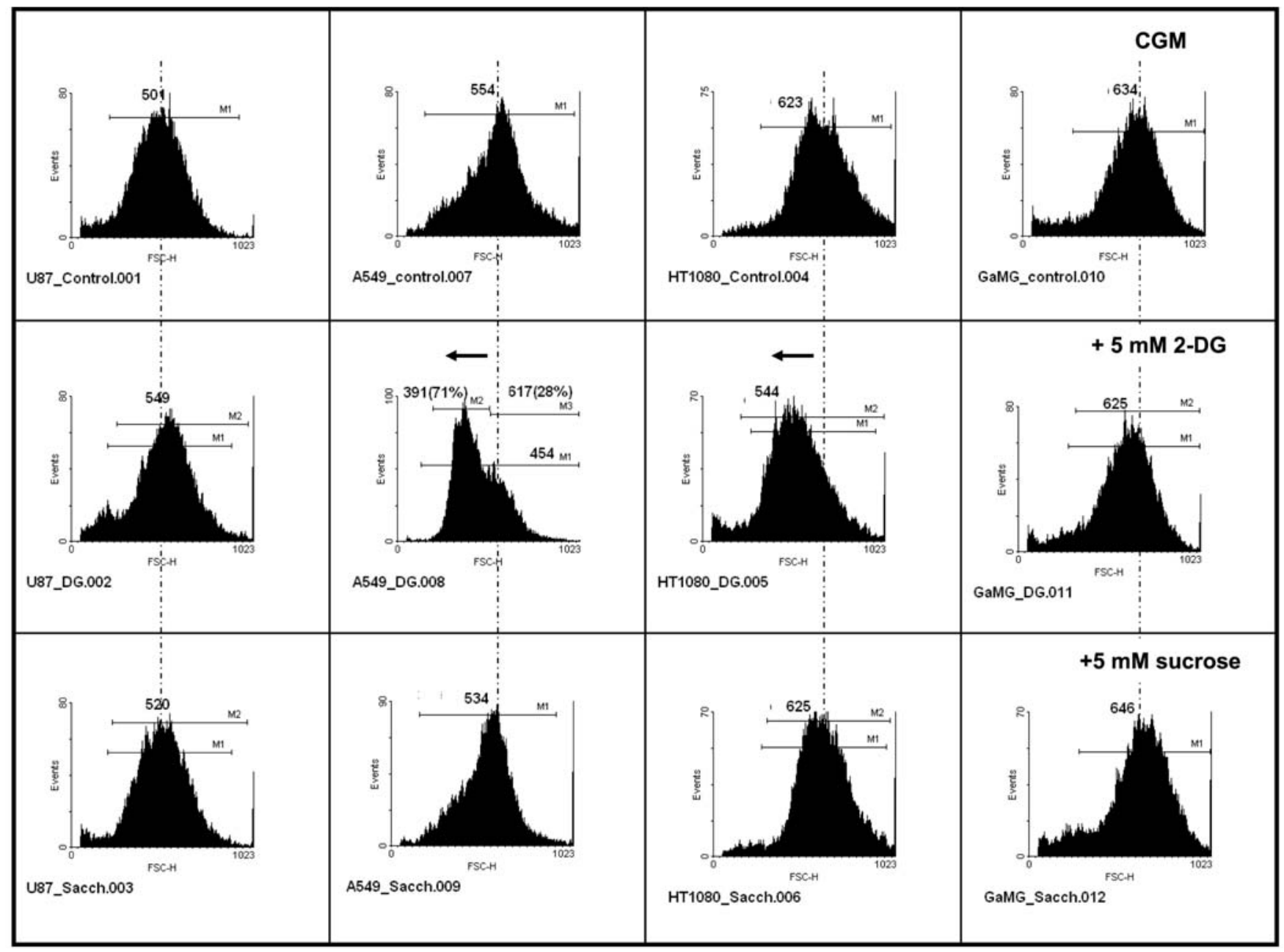

Figure 1. Representative histograms showing relative changes in cell size detected by forward light scatter (FSC) flow cytometry in four tumor cell lines after a 24-h incubation in standard CGM (controls and top histograms) and in CGM supplemented with 5 mM 2DG (middle histograms) or 5 mM sucrose (bottom histograms). The modal FSC values in the control samples are indicated by dashed lines.

appearance of two subpopulations of A549 cells. The first subpopulation ( $70 \%$ of cells) exhibited lower FSC signals (391 a.u.) than the control cells (554 a.u.). Cells in the second subpopulation were larger (617 a.u.) than untreated A549 cells.
The size of all tested tumor cells remained unchanged upon long-term exposure to $5 \mathrm{mM}$ of sucrose. Neither 2DG nor sucrose ( $5 \mathrm{mM}, 24 \mathrm{~h}$ ) induced any notable changes in the cell cycle distribution of the four cell lines tested (data not shown). 

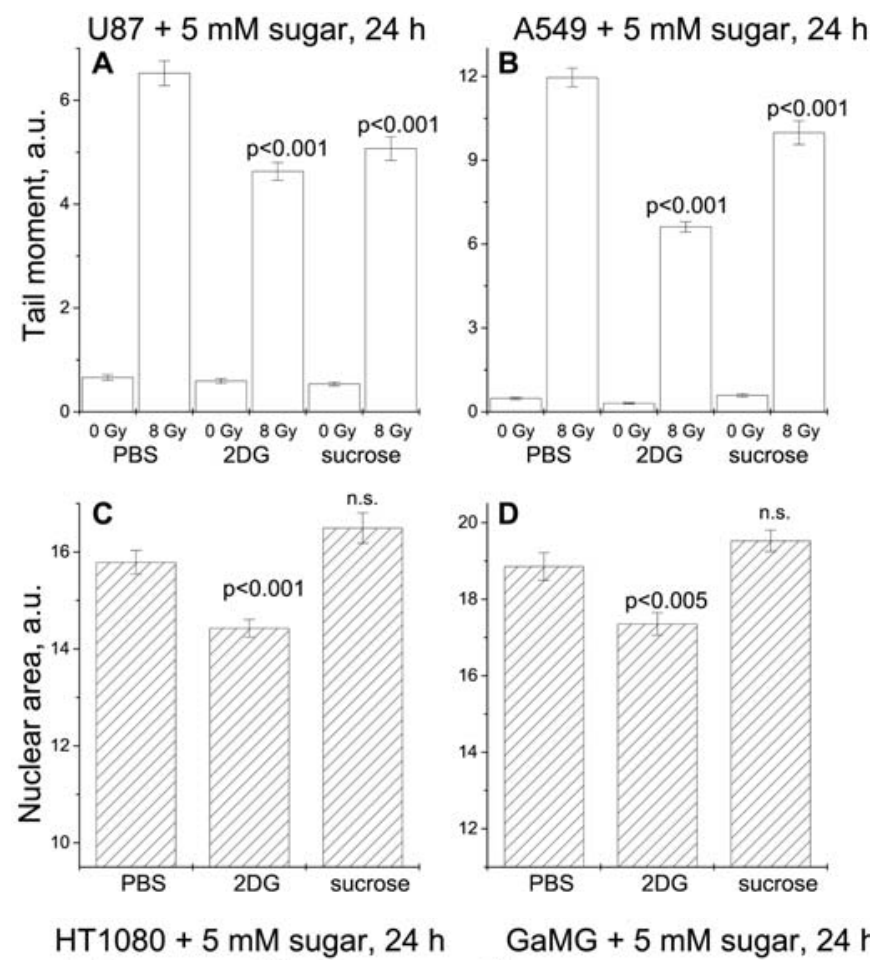

GaMG + 5 mM sugar, 24 h
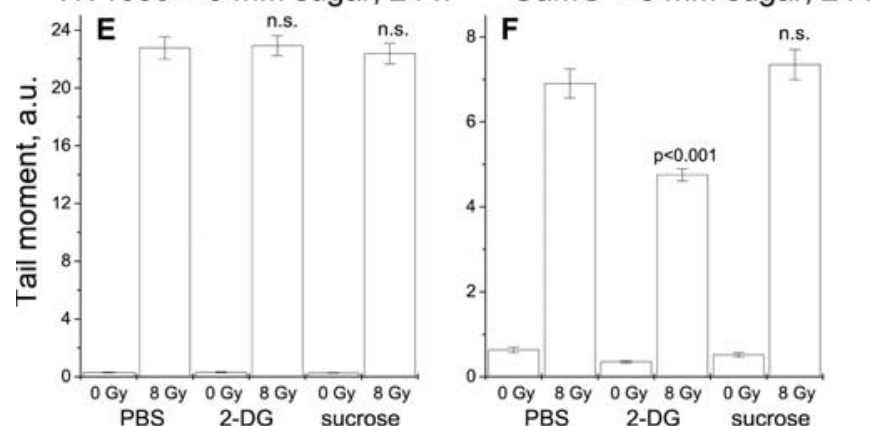

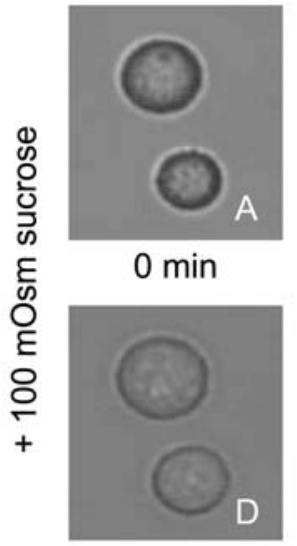

7 min

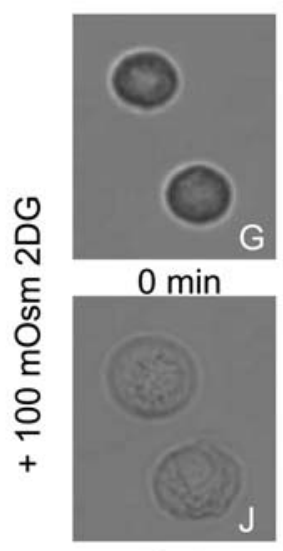

7 min

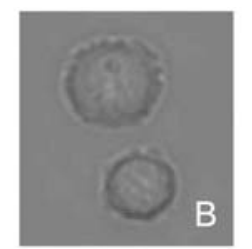

$1 \mathrm{~min}$

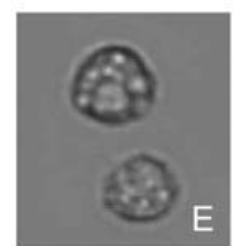

$13 \mathrm{~min}$

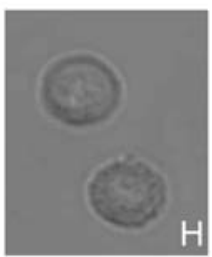

$1 \mathrm{~min}$

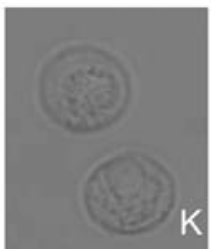

$13 \mathrm{~min}$

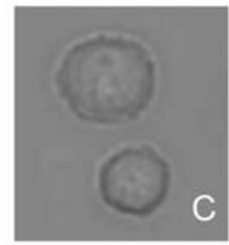

2 min

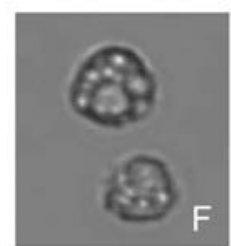

20 min

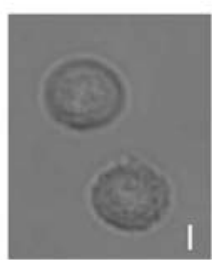

$2 \min$

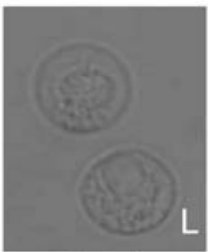

$20 \mathrm{~min}$

Figure 3. Volume changes in A549 cells exposed to hypotonic 100-mOsm medium containing sucrose (A-F) or $2 \mathrm{DG}(\mathrm{G}-\mathrm{L})$ as the major solute. A and $\mathrm{G}$ show cells in isotonic CGM (time $\leq 0, \sim 300 \mathrm{mOsm}$ ). Hypotonic sucrose caused rapid cell swelling to the maximum volume within the first 2 min ( $\mathrm{B}$ and $\mathrm{C}$ ). Subsequently, the cells gradually shrank over the next 20 min (E and F). In contrast, the hypotonic 2DG solution resulted in continuous swelling over the entire observation period (H-L).
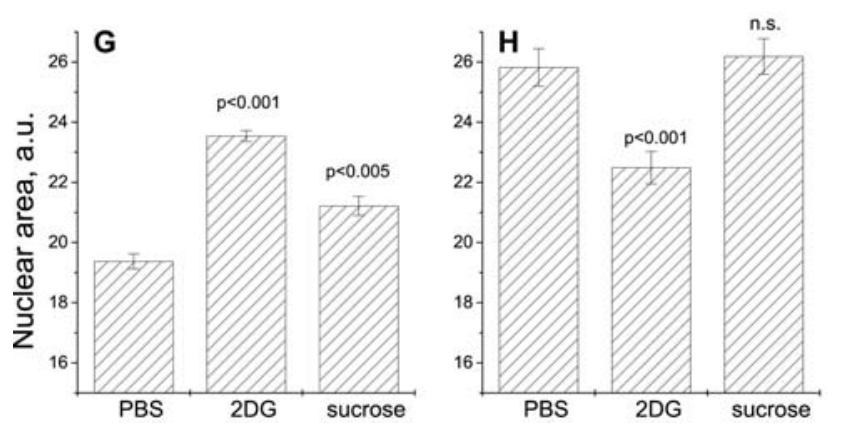

Figure 2. Comet parameters of U87 (A and C), A549 (B and D), HT1080 (E and $\mathrm{G})$ and GaMG (F and H) cells. Each cell line was incubated for $24 \mathrm{~h}$ in CGM containing $5 \mathrm{mM}$ of 2DG (middle bars) or sucrose (right-hand bars). In A, B, E and F, the initial Comet assay Tail Moment data without irradiation and after irradiation with $8 \mathrm{~Gy}$ in vitro are shown. Corresponding data for the nuclear area in non-irradiated samples are shown in C, D, G and H. Each bar graph represents the mean \pm SE of 75-100 cells. Statistical comparisons refer to sugar-free controls.

DNA fragmentation caused by long-term sugar treatment ( $5 \mathrm{mM}, 24 \mathrm{~h})$ and irradiation. Each cell line was subjected to $5 \mathrm{mM}$ of sugar for $24 \mathrm{~h}$ prior to irradiation. DNA fragmentation was assessed from the initial Comet assay TM measured immediately after irradiation with 8 Gy (Fig. 2A, B, E and F). Nuclear area data acquired from non-irradiated cell samples in the same runs of the Comet assay are presented in Fig. 2C, D, $\mathrm{G}$ and $\mathrm{H}$.

Long-term pretreatment with 2DG led to a significant TM decrease in irradiated U87 cells, from $\sim 7$ a.u. (control) to $\sim 4.5$ a.u. (Fig. 2A). Sucrose reduced TM to a lesser extent ( $\sim 5$ a.u.). 2DG-mediated protection against IR-induced DNA fragmentation was associated with a significant reduction in the nuclear area of non-irradiated U87 cells. The effects of the sugars on IR-induced DNA fragmentation and nuclear area in the A549 and GaMG cell lines were qualitatively similar to those of U87 cells. In contrast, both sugars markedly increased the nuclear area of HT1080 cells, without affecting TM values.

The Comet assay did not reveal any enhancement of IRinduced DNA fragmentation in tumor cells pretreated with 2DG. Contrary to expectation, 2DG afforded strong protection against IR-induced DNA damage in three of the four cell lines tested (U87, A549 and GaMG). Sucrose decreased IR-induced DNA fragmentation in two cell lines (U87 and A549).

The possibility cannot be excluded that 2DG failed to enhance IR-induced DNA fragmentation due to the low intracellular concentrations of 2DG present during irradiation. In order to ensure substantial cytosolic concentrations of 2DG, we further explored the possibility of the intracellular delivery of 2DG through swelling-activated channels in the plasma membrane of tumor cells. 
relative cell volume, $v$
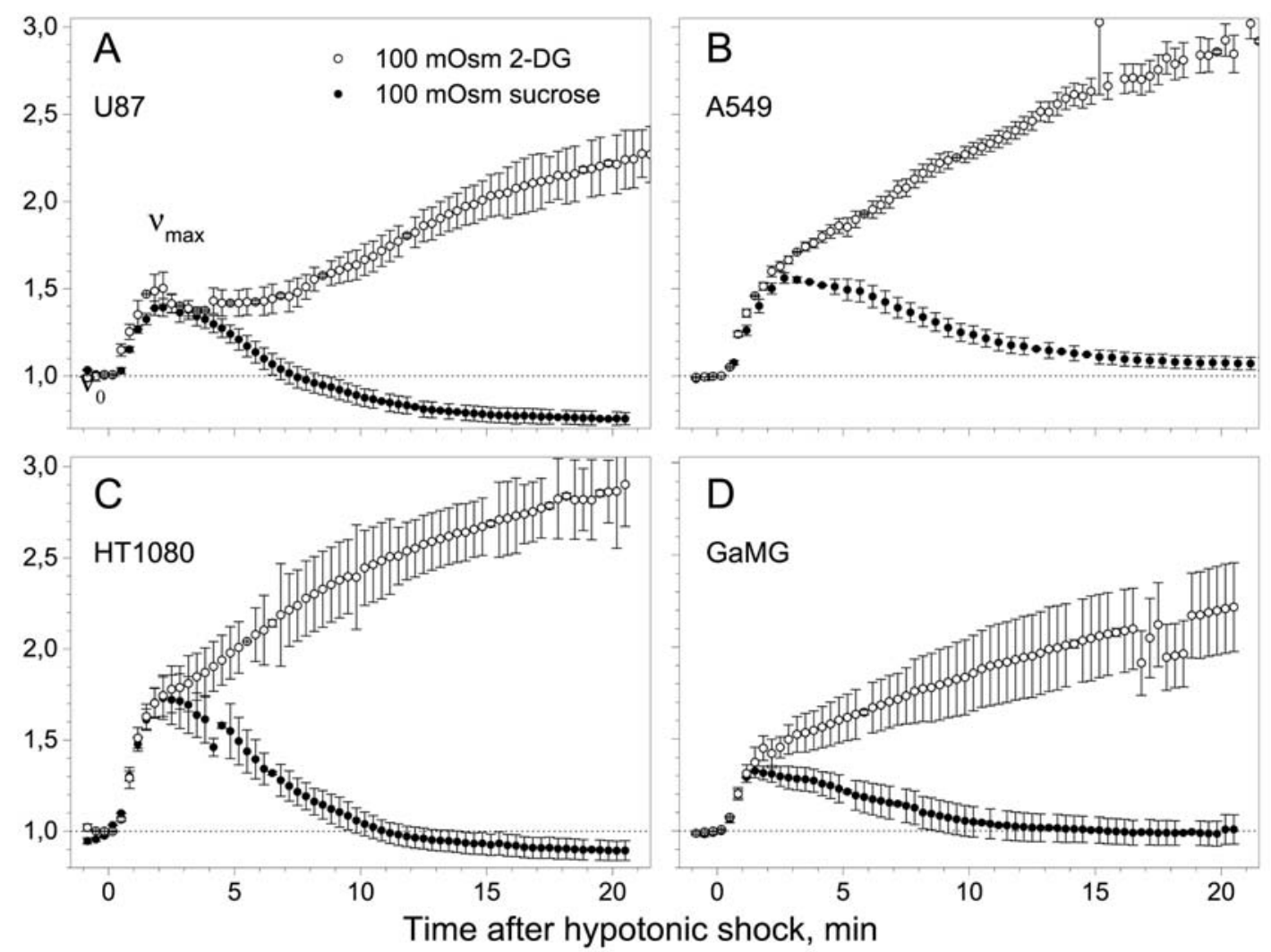

Figure 4. Relative cell volume changes of the indicated tumor lines in hypotonic 100-mOsm solutions containing 2DG (empty circles) or sucrose (filled circles) as the major solute. Cells were originally exposed to isotonic CGM, which was replaced at zero time by a hypotonic sugar solution. After initial swelling, the cells exhibited RVD in hypotonic sucrose medium. In contrast, 2DG induced secondary swelling in the tested cell lines. Each data point is the mean \pm SE of 5-9 cells.

Cell volumetry in hypotonic sugar-substituted media. Cell volume changes were monitored by videomicroscopy following the rapid transfer of cells from isotonic CGM ( 300 mOsm) to strongly hypotonic 100 -mOsm solutions containing sucrose or 2DG as the major solute. As seen in Fig. 3A-F, 100-mOsm sucrose caused a biphasic cell volume response in A549 cells. The cells first swelled rapidly within $\sim 1-2$ min, then shrank slowly over the following 5-20 min. In contrast, the application of 100-mOsm 2DG resulted in continuous cell swelling over the entire observation period (Fig. 3G-L).

Fig. 4 shows the mean volumetric response of the four cell lines to the 100-mOsm sugar solution. Data were derived from microphotographs, such as those shown in Fig. 3. In the presence of one of the sugars (2DG or sucrose), the cell lines exhibited qualitatively similar transient volumetric responses with respect to both initial and secondary volume changes.

Independent of the sugar used, the sudden exposure to hypotonicity caused all the tumor cell types to swell rapidly within the first $2 \mathrm{~min}$ from their original isotonic volume $v_{0}$ to the $v_{\max }$ level due to the fast influx of water. The two sugars resulted in qualitatively similar rates and magnitudes of initial swelling in a particular cell line. Although the same osmotic shift $(300 \rightarrow 100 \mathrm{mOsm})$ was applied, the initial swelling of fibrosarcoma HT1080 cells ( $\left.v_{\max } \approx 1.75\right)$ was markedly increased compared to the swelling observed in lung carcinoma A549 cells $(\sim 1.55)$ and in the two glioblastoma cell lines, U87 and GaMG (1.3-1.4).
The data in Fig. 4 reveal striking differences between sucrose and 2DG in terms of their effects on secondary cell volume changes. After the fast initial swelling in hypotonic sucrose-substituted medium, all the cell types underwent a regulatory volume decrease (RVD), during which they shrank gradually, recovering their original isotonic volume $\left(v_{0}\right)$ within 10-15 min despite persisting hypotonicity. RVD involves the release of cytosolic solutes through swelling-activated membrane pathways along with osmotically-driven water loss, which allows the cells to recover their original isotonic volume $(19,26,27)$. In agreement with our findings for two glioblastoma lines (U87 and GaMG), other glioma cells (including the D54-MG line and primary glioma cells from patient biopsies) are able to quickly readjust their volume in anisotonic media via several mechanisms, including chloride pathways and water channels (aquaporins) $(28,29)$.

In sharp contrast to disaccharide sucrose, the monomeric sugar 2DG not only completely abolished RVD, but even induced secondary swelling in the tested cell lines (Fig. 4). As discussed below, RVD inhibition is based on the rapid influx of 2DG from the extracellular medium into the cytosol. Of note, the tumor cell membranes were impermeable to disaccharide sucrose, as evidenced by RVD in sucrose-substituted medium.

Hypotonic treatment of cells by membrane-impermeable sucrose did not result in any significant changes in the proliferation activity of the four cell lines (Table I), and hypotonic 


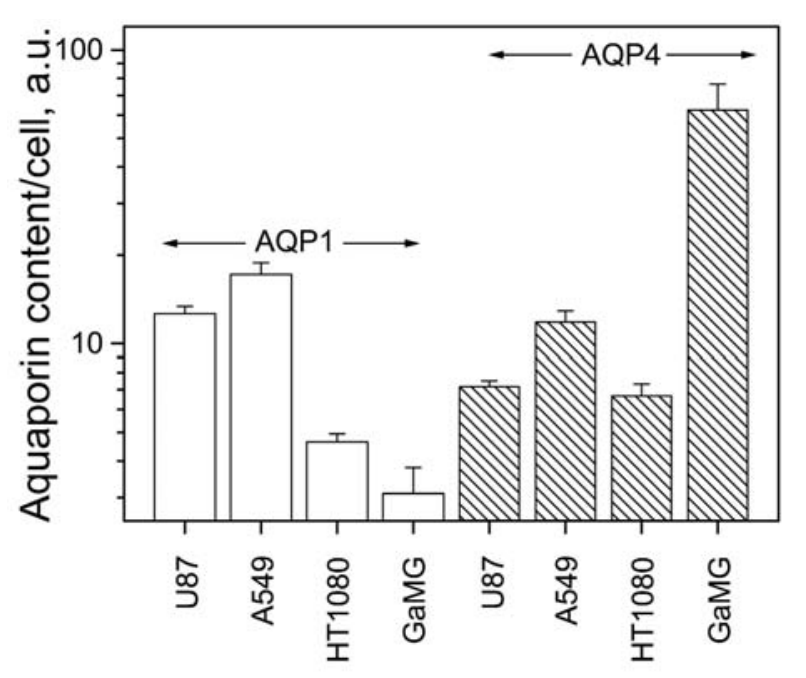

Figure 5. Expression of AQP1 and AQP4 in four tumor cell lines. Data (mean \pm SD of 50-75 cells) were derived from fluorescence photographs, such as those shown in Figs. 5 and 6.

cell loading with 2DG did not significantly affect the growth of the two glioblastoma lines (U87 and GaMG), both of which exhibited relatively small secondary swelling (Fig. 4). Growth inhibition of 23-24\% in A549 and HT1080 cells (Table I) was associated with their extreme secondary swelling in hypotonic 2DG-substituted solution (Fig. 4B and C)

Expression of aquaporins in tumor cell lines. Hypotonic cell swelling is governed by the water permeability of cell membranes, among other parameters $(19,29)$. In order to account for the varying volume response of the tumor cells to hypotonicity, we further analyzed the expression of the aquaporins AQP1 and AQP4.

Fluorescent images of the tumor cells immunostained with anti-AQP1 and anti-AQP4 antibodies were analyzed for each cell line and each aquaporin, and are summarized in Fig. 5. As shown, the expression of AQP1 decreased in the order: A549 > U87 > HT1080 > GaMG, whereas a nearly reverse order was obtained in the case of AQP4: GaMG > A549 > $\mathrm{U} 87 \approx \mathrm{HT} 1080$. Notably, GaMG cells exhibited the highest expression of AQP4, but the lowest expression of AQP1. Western blot analysis yielded qualitatively similar results for aquaporin expression (data not shown).

A comparison of the volumetric and immunostaining data failed to reveal any relationship between aquaporin expression and the swelling behavior of the tumor cells. Thus, despite the relatively poor expression of the aquaporins, HT1080 cells exhibited the largest volume changes in response to hypotonic solutions of the sugars.

IR-induced DNA fragmentation in hypotonically-treated tumor cells. Tumor cells were preloaded hypotonically with 2DG or treated with hypotonic sucrose for $\sim 20 \mathrm{~min}$. Cells were then diluted (1:9) with an agarose solution containing $100 \mathrm{mM}$ of 2DG or sucrose irradiated with $8 \mathrm{~Gy}$ and analyzed for IRinduced DNA fragmentation by the Comet assay. Despite the strong difference between the intracellular concentration of 2DG and sucrose during irradiation, the effects of two sugars were similar in three of the four cell lines tested.
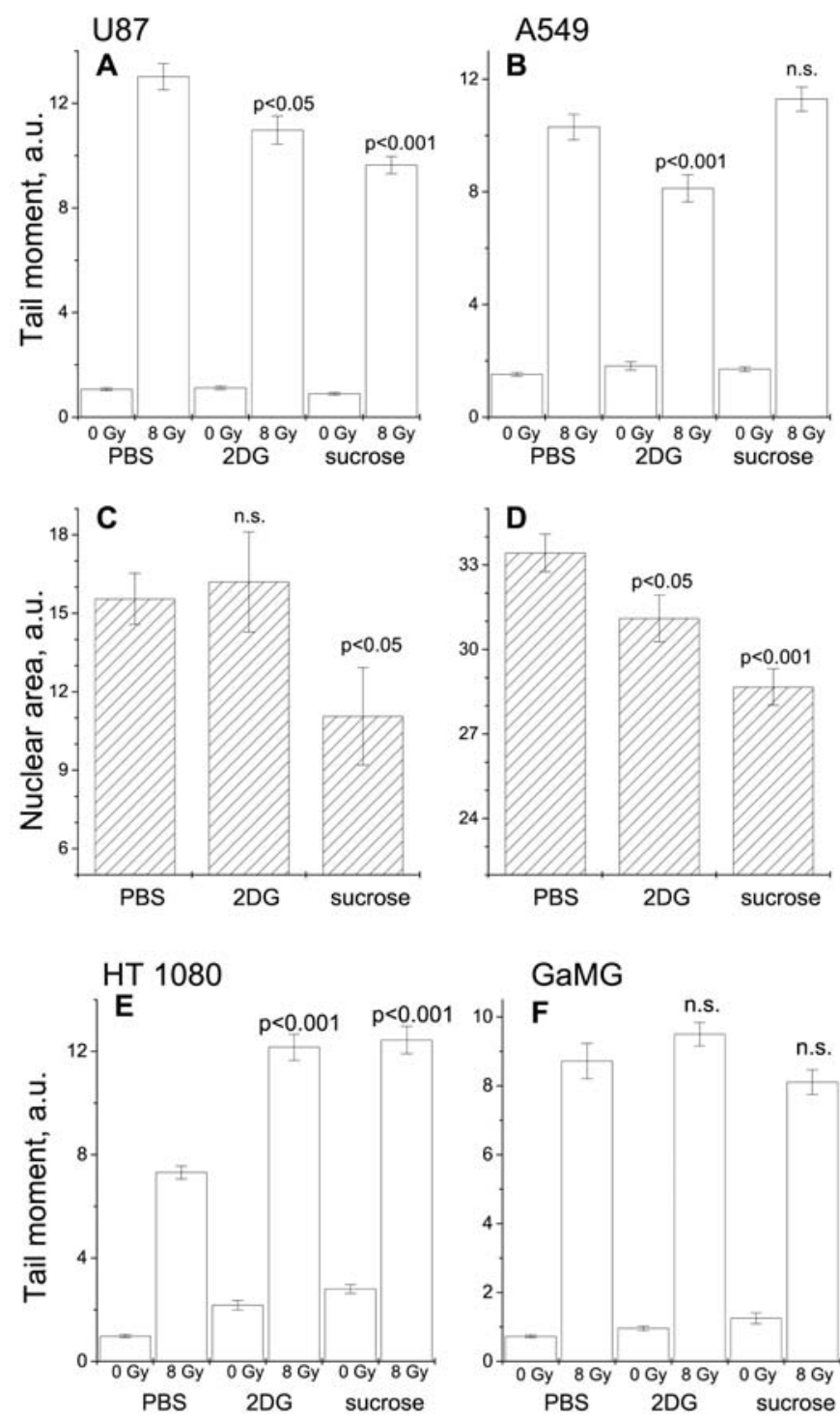

GaMG
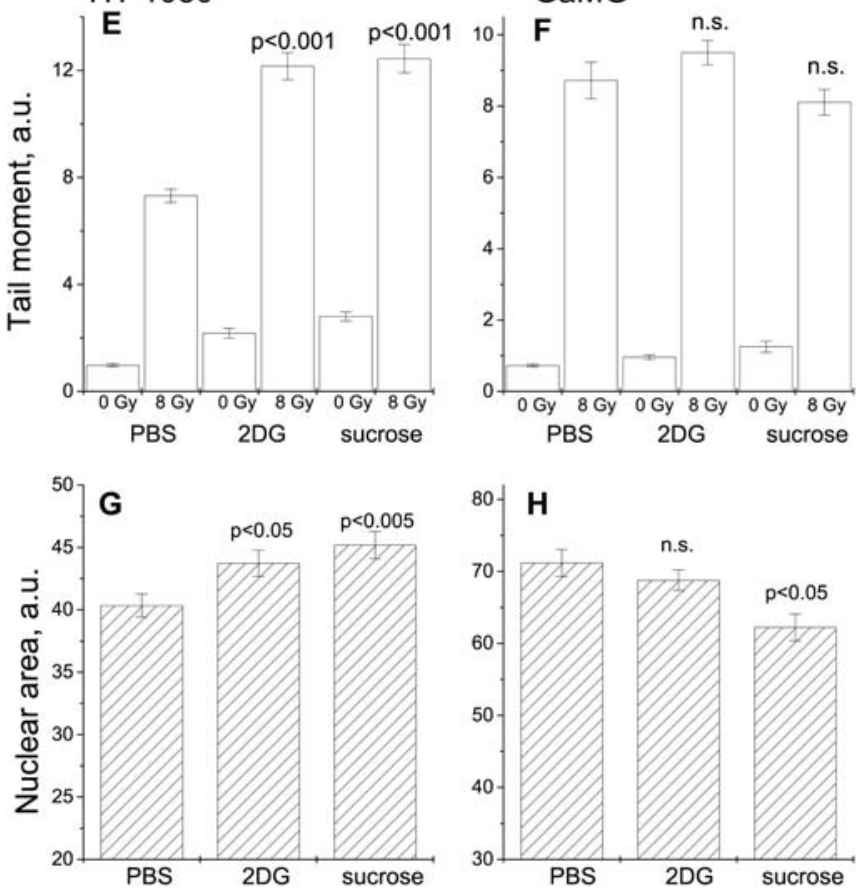

Figure 6. Comparison of the Comet assay parameters for the indicated cell lines treated with hypotonic $2 \mathrm{DG}$ or sucrose of the same osmolality of 100 mOsm. For further details see Fig. 2 legend.

Hypotonic loading with 2DG exerted very different effects on IR-induced DNA fragmentation in the four cell lines, ranging from moderate protection in U87 and A549 cells (Fig. 6A and B) to a strong increase in DNA fragmentation in HT1080 cells (Fig. 6E) and no effect in GaMG cells (Fig. 6F), compared to isotonic conditions (PBS). The TM data obtained with hypotonic sucrose matched the corresponding data of 2DG-treated cells, with the exception of A549 cells, in which DNA fragmentation was reduced by $2 \mathrm{DG}$, but unaffected by sucrose (Fig. 6B). 
Interestingly, the increase in IR-induced DNA fragmentation in HT1080 cells (Fig. 6E) correlated with the expansion of the nuclear area caused by hypotonic sugar solutions in non-irradiated samples (Fig. 6G). Likewise, sucrose-mediated protection of DNA against irradiation in U87 cells (Fig. 6A) was associated with a significant reduction in the size of the nucleus (Fig. 6C). Notably, the strong decrease in the nuclear area of sucrose-treated U87 cells revealed by the Comet assay (Fig. 6C) is in good agreement with the marked secondary shrinkage of these cells via the RVD mechanism in hypotonic sucrose solutions observed in independent volumetric experiments (Fig. 4A).

\section{Discussion}

The two methods used for loading tumor cells with 2-deoxyD-glucose (2DG) yielded consistent results regarding the cell type-specific toxicity of this sugar (Table I). Irrespective of the loading method, 2DG inhibited the growth of HT1080 and A549 cells. Moreover, the long-term incubation of these two cell lines by 2DG was correlated with a marked decrease in cell size revealed by flow cytometry (Fig. 1). In contrast, both glioblastoma cell lines (U87 and GaMG) appeared to be resistant to 2DG, as neither long-term nor short-term treatment with 2DG significantly affected the growth and cell size distribution of these cells (Table I, Fig. 1). Irrespective of the treatment method, sucrose did not exert any detrimental effects on the proliferative ability of any of the tested cell lines (Table I). This was apparently due to the poor membrane permeability to this disaccharide (see below).

Contrary to expectations raised by previous studies (4-6), the long-term incubation of tumor cells with 2DG did not result in any enhancement of IR-induced DNA fragmentation (Fig. 2). Instead, the opposite effect was observed; i.e., a significant reduction in DNA fragmentation after irradiation occurred in three of the four cell lines grown in 2DG-supplemented media. It is noteworthy that in the 2DG-responsive tumor lines (U87, A549 and GaMG), the reduction in IRinduced DNA damage correlated with a marked 2DG-mediated decrease in the nuclear area of non-irradiated samples (Fig. 2C, $\mathrm{D}$ and $\mathrm{H})$. Consequently, the observed radioprotection of DNA by 2 DG in these three tumor lines may have been a secondary effect of the shrinkage of the nucleus (via chromatin condensation).

The lack of the expected enhancement of IR-induced DNA fragmentation in tumor cells grown with $5 \mathrm{mM} 2 \mathrm{DG}$ can also be attributed to the poor uptake of this sugar by tumor cells. In order to ensure substantial cytosolic concentrations of 2DG during irradiation, we exploited the possibility of the rapid intracellular delivery of this monomeric sugar through swelling-activated membrane channels in hypotonically swollen tumor cells. Swelling-activated anion channels with high permeability to small organic osmolytes, including monomeric sugars, polyols and amino acids, are ubiquitously expressed in mammalian cells $(26,30,31)$ According to previous studies $(19,31)$, the varying effects of $2 \mathrm{DG}$ and sucrose on the secondary volume response of tumor cells observed here (Fig. 4) can be explained by the ability of the swelling-activated channels to selectively conduct the small monomeric sugar 2DG while remaining impermeable to the larger disaccharide sucrose. Driven by the inwardly directed gradient, the influx of 2DG abolished the regulatory volume decrease (RVD) by compensating for the efflux of intracellular solutes. The observed inhibition of RVD implies a substantial uptake of 2DG by tumor cells. Earlier studies justify the use of RVD inhibition as an indicator of swelling-activated membrane permeability for small extracellular solutes $(19,32)$. Moreover, the secondary swelling observed in the tested cell lines (Fig. 4) suggests that the rate of 2DG influx into tumor cells was even larger than that of cytosolic solute efflux.

Despite the avid hypotonic uptake of 2DG by all the tumor cell lines (Fig. 4), only HT1080 cells exhibited a significant increase in IR-induced DNA fragmentation upon loading with 2DG (Fig. 6E). Interestingly, these particular cells underwent the strongest secondary swelling in hypotonic 2DG medium (Fig. 4C). Nevertheless, enhanced DNA fragmentation in HT1080 cells can hardly be attributed to radiosensitization by intracellular 2DG. As evidenced by Fig. 8E, not only 2DG but also the membrane-impermeable sucrose markedly increased IR-induced DNA fragmentation in HT1080 cells after hypotonic treatment. Moreover, irrespective of sugar composition, hypotonic treatment induced a marked expansion of the nuclear area in non-irradiated HT1080 cells (Fig. 6G). These data suggest that hypotonicity-mediated decondensation of chromatin may be responsible for the observed enhancement of IR-induced DNA fragmentation in this particular tumor cell line. In agreement with this interpretation, the decrease in nuclear area of hypotonically-treated U87 and A549 cells was correlated with a significant protection against IR-induced DNA fragmentation detected by the Comet assay (Fig. 6A-D). Consequently, depending on cell type and extracellular sugar composition, hypotonicity can induce the compaction or expansion of DNA/chromatin, leading, respectively, to a decrease or increase in IR-induced DNA fragmentation. These tendencies are in good agreement with the results of a recent study (33), in which several radioresistent cell lines exhibited a highly condensed chromatin texture, while cellular radiosensitivity was correlated with a relaxed chromatin conformation.

In conclusion, in all the tumor cell lines tested, hypotonic cell swelling led to the activation of endogenous volumesensitive channels, which rendered the cell membranes highly permeable to the monosaccharide 2-deoxy-D-glucose (but not to the disaccharide sucrose). Although the present study focused on the hypotonic loading of cells with 2DG, the approach can be generally applied for the rapid introduction of large amounts of various non-native monomeric sugars and other small molecules as potential radiosensitizers/-protectors into a variety of malignant and normal cells of interest. We also demonstrated that isotonic and hypotonic sugar solutions lead to persisting alterations in chromatin structure in nonirradiated tumor cells, as revealed by nuclear area analysis. Depending on cell type, either chromatin expansion or condensation is induced by sugar treatment, which may in turn be responsible for the observed enhancement or reduction of radiation-induced DNA fragmentation measured by the Comet assay. These results raise interesting questions for further studies on the mechanistic links between the sugarmodulated cell volume changes, chromatin structure and radiosensitivity of tumor and normal cells. 


\section{References}

1. Warburg O: On the origin of cancer cells. Science 12: 309-314, 1956.

2. Brown J: Effects of 2-deoxyglucose on carbohydrate metablism: review of the literature and studies in the rat. Metabolism 11: 1098-1112, 1962.

3. Hunter AJ and Blekkenhorst GH: The effects of 2-deoxyglucose and amino-oxyacetic acid on the radiation response of mammalian cells in vitro. Int J Radiat Biol 73: 311-324, 1998.

4. Varshney R, Dwarakanath B and Jain V: Radiosensitization by 6-aminonicotinamide and 2-deoxy-D-glucose in human cancer cells. Int J Radiat Biol 81: 397-408, 2005.

5. Coleman MC, Asbury CR, Daniels D, Du J, Aykin-Burns N, Smith BJ, Li L, Spitz DR and Cullen JJ: 2-deoxy-D-glucose causes cytotoxicity, oxidative stress, and radiosensitization in pancreatic cancer. Free Radic Biol Med 44: 322-331, 2008.

6. Khaitan D, Chandna S, Arya MB and Dwarakanath BS: Differential mechanisms of radiosensitization by 2-deoxy-Dglucose in the monolayers and multicellular spheroids of a human glioma cell line. Cancer Biol Ther 5: 1142-1151, 2006.

7. Dwarakanath BS, Khaitan D and Ravindranath T: 2-deoxyD-glucose enhances the cytotoxicity of topoisomerase inhibitors in human tumor cell lines. Cancer Biol Ther 3: 864-870, 2004.

8. Simons AL, Fath MA, Mattson DM, Smith BJ, Walsh SA, Graham MM, Hichwa RD, Buatti JM, Dornfeld K and Spitz DR: Enhanced response of human head and neck cancer xenograft tumors to cisplatin combined with 2-deoxy-D-glucose correlates with increased 18F-FDG uptake as determined by PET imaging. Int J Radiat Oncol Biol Phys 69: 1222-1230, 2007.

9. Mohanti BK, Rath GK, Anantha N, Kannan V, Das BS, Chandramouli BA, Banerjee AK, Das S, Jena A, Ravichandran R, Sahi UP, Kumar R, Kapoor N, Kalia VK, Dwarakanath BS and Jain V: Improving cancer radiotherapy with 2-deoxy-D-glucose: phase I/II clinical trials on human cerebral gliomas. Int J Radiat Oncol Biol Phys 35: 103-111, 1996.

10. Singh D, Banerji AK, Dwarakanath BS, Tripathi RP, Gupta JP, Mathew TL, Ravindranath T and Jain V: Optimizing cancer radiotherapy with 2-deoxy-d-glucose dose escalation studies in patients with glioblastoma multiforme. Strahlenther Onkol 181: 507-514, 2005

11. Andringa KK, Coleman MC, Aykin-Burns N, Hitchler MJ, Walsh SA, Domann FE and Spitz DR: Inhibition of glutamate cysteine ligase activity sensitizes human breast cancer cells to the toxicity of 2-deoxy-D-glucose. Cancer Res 66: 1605-1610, 2006.

12. Maher JC, Krishan A and Lampidis TJ: Greater cell cycle inhibition and cytotoxicity induced by 2-deoxy-D-glucose in tumor cells treated under hypoxic vs aerobic conditions. Cancer Chemother Pharmacol 53: 116-122, 2004.

13. Sridhar R, Stroude EC and Inch WR: Cytotoxicity of glucose analogues in V79 multicell spheroids. In Vitro 15: 685-690, 1979.

14. Kern KA and Norton JA: Inhibition of established rat fibrosarcoma growth by the glucose antagonist 2-deoxy-D-glucose. Surgery 102: 380-385, 1987.

15. Tannock IF, Guttman P and Rauth AM: Failure of 2-deoxy-Dglucose and 5-thio-D-glucose to kill hypoxic cells of two murine tumors. Cancer Res 43: 980-983, 1983.

16. Bala $\mathrm{M}$ and Goel HC: Modification of low dose radiation induced radioresistance by 2-deoxy-D-glucose in Saccharomyces cerevisiae: mechanistic aspects. J Radiat Res 48: 335-346, 2007.
17. Dwarkanath BS, Zolzer F, Chandana S, Bauch T, Adhikari JS, Muller WU, Streffer C and Jain V: Heterogeneity in 2-deoxy-Dglucose-induced modifications in energetics and radiation responses of human tumor cell lines. Int J Radiat Oncol Biol Phys 50: 1051-1061, 2001.

18. Kurtoglu M, Gao N, Shang J, Maher JC, Lehrman MA, Wangpaichitr M, Savaraj N, Lane AN and Lampidis TJ: Under normoxia, 2-deoxy-D-glucose elicits cell death in select tumor types not by inhibition of glycolysis but by interfering with $\mathrm{N}$ linked glycosylation. Mol Cancer Ther 6: 3049-3058, 2007.

19. Reuss R, Ludwig J, Shirakashi R, Ehrhart F, Zimmermann H, Schneider S, Weber MM, Zimmermann U, Schneider H and Sukhorukov VL: Intracellular delivery of carbohydrates into mammalian cells through swelling-activated pathways. J Membr Biol 200: 67-81, 2004.

20. Djuzenova CS, Güttler T, Berger S, Katzer A and Flentje M: Differential response of human glioblastoma cell lines to combined camptothecin and ionizing radiation treatment. Cancer Biol Ther 7: 364-373, 2008.

21. Djuzenova CS, Mühl B, Fehn M, Oppitz U, Müller B and Flentje M: Radiosensitivity in breast cancer assessed by the Comet and micronucleus assays. Br J Cancer 94: 1194-1203, 2006.

22. Bürger S, Schindler D, Fehn M, Mühl B, Mahrhofer H, Flentje M, Hoehn H, Seemanová E and Djuzenova CS: Radiation-induced DNA damage and repair in peripheral blood mononuclear cells from Nijmegen breakage syndrome patients and carriers assessed by the Comet assay. Environ Mol Mutagen 47: 260-270, 2006.

23. Olive PL, Banáth JP and Durand RE: Heterogeneity in radiationinduced DNA damage and repair in tumor and normal cells measured using the 'comet' assay. Radiat Res 122: 86-94, 1990.

24. Djuzenova C, Mühl B, Schakowski R, Oppitz U and Flentje M: Normal expression of DNA repair proteins, hMre11, $\operatorname{Rad} 50$ and Rad51 but protracted formation of Rad50 containing foci in Xirradiated skin fibroblasts from radiosensitive cancer patients. Br J Cancer 90: 2356-2363, 2004.

25. Aft RL, Zhang FW and Gius D: Evaluation of 2-deoxy-Dglucose as a chemotherapeutic agent: mechanism of cell death. Br J Cancer 87: 805-812, 2002.

26. Lang F, Busch GL and Völkl H: The diversity of volume regulatory mechanisms. Cell Physiol Biochem 8: 1-45, 1998.

27. Lange K: Regulation of cell volume via microvillar ion channels. J Cell Physiol 185: 21-35, 2000

28. Ernest NJ, Weaver AK, van Duyn LB and Sontheimer HW: Relative contribution of chloride channels and transporters to regulatory volume decrease in human glioma cells. Am J Physiol Cell Physiol 288: C1451-C1460, 2005.

29. McCoy E and Sontheimer H: Expression and function of water channels (aquaporins) in migrating malignant astrocytes. Glia 55: 1034-1043, 2007

30. Eggermont J, Trouet D, Carton I and Nilius B: Cellular function and control of volume-regulated anion channels. Cell Biochem Biophys 35: 263-274, 2001.

31. Sukhorukov VL, Reuss R, Zimmermann D, Held C, Müller KJ Kiesel M, Gessner P, Steinbach A, Schenk WA, Bamberg E and Zimmermann U: Surviving high-intensity field pulses: strategies for improving robustness and performance of electrotransfection and electrofusion. J Membr Biol 206: 187-201, 2005.

32. Pasantes-Morales H, Murray RA, Sánchez-Olea R and Morán J: Regulatory volume decrease in cultured astrocytes: 2. Permeability pathway to amino-acids and polyols. Am J Physiol 266: C172-C178, 1994.

33. Rajab NF, McKenna DJ, Diamond J, Williamson K, Hamilton PW and McKelvey-Martin VJ: Prediction of radiosensitivity in human bladder cell lines using nuclear chromatin phenotype. Cytometry 69: A1077-A1085, 2006. 